\title{
Are So-Called Cancer-Testis Genes Expressed Only in Testis?
}

\author{
Soudeh Ghafouri-Fard*, Fatemeh Rezazadeh, Davood Zare-Abdollahi, Mir \\ Davood Omrani, Abolfazl Movafagh
}

\begin{abstract}
Cancer-testis (CT) antigens are a group of tumor-associated antigens with restricted expression in normal tissues except for testis and expression in a wide variety of tumor tissues. This pattern of expression makes them suitable targets for immunotherapy as well as potential biomarkers for early detection of cancer. However, some genes attributed to this family are now known to be expressed in other normal tissues which put their potential applications in immunotherapy and cancer detection under question. Here we analyzed expression of two previously known CT antigens, RHOXF2 and PIWIL2, in AML patients versus normal donors and found no significant difference in the expression of these genes between the two groups. As these two genes showed expression in normal leukocytes, their expression pattern seems to be wider than to be attributed to the CT gene family. Future research should focus on the expression profiles of so called CT antigens to find those with more testis specific expression.
\end{abstract}

Keywords: Cancer-testis antigen - RHOXF2 - PIWIL2 - leukocytes - specificity of expression

Asian Pac J Cancer Prev, 15 (18), 7703-7705

\section{Introduction}

Cancer-testis (CT) antigens with a restricted expression pattern in normal tissues except testis and expression in a wide variety of tumors are promising targets for immunotherapy (Ghafouri-Fard and Modarressi, 2009). In a bioinformatics based search in NCBI and UniGene, we selected two testis-specific genes: Rhox homeobox family, member 2 (RHOXF2, PEPP2) and Piwi-like RNAmediated gene silencing 2 (PIWIL2) according to their restricted expression in normal testis, putative function in carcinogenesis process and abnormal elevated expression in different cancers.

RHOXF2 is a newly found CT gene and a stem cell marker (Ghafouri-Fard et al., 2012). The membraneassociated RHOXF2 has been shown to be involved in cell to cell contacts. Its over expression has increased membrane microviscosity, implying a potential role for it in regulation of membrane and microtubules (Zou and Zhong, 2012). It also functions as a transcriptional repressor. Transduction of RHOXF2 has been shown to make HF6 (a mouse bone marrow derived cell line) factor-independent, while its knockdown has growth inhibitory effect in HGC27 gastric cancer cell line. Additionally, transplantation of RHOXF2-transduced HF6 cells into irradiated mice has rapidly induced leukemia. Furthermore, $R H O X F 2$ has been shown to be expressed in some human hematopoietic cell lines including immature leukemic cell lines TF-1 and K562 and a range of human cancer samples (Shibata-Minoshima et al., 2012).

PIWIL2 is a germline stem cell gene whose ectopic expression is associated with cancer stem cell development. Expression analysis has shown that it is absolutely expressed in the germline stem cell of testis but not in the adult tissue stem cells and somatic cells. It has been shown to be involved in DNA repair via chromatin relaxation, so it has been suggested as a gatekeeper against DNA damage-mediated carcinogenesis (Yin et al., 2011). PIWIL2 expression has been shown to be linked with increased proliferation and apoptosis inhibition (Lee et al., 2006). Its expression has been detected in a wide variety of primary cancers and tumor cell lines including Jurkat leukemia cell line. PIWIL2 knock down with small interference RNA has suppressed Stat 3 and Bcl-XL expression and stimulated apoptosis (Lee et al., 2006). In a previous study of PIWIL2 expression in AML patients, no significant changes have been detected in the expression pattern between patients and healthy donors except for down regulation of PIWIL2 in 5 AML patients and up regulation in 3 AML and 3 myelodysplastic syndrome patients (Yazarloo et al., 2013).

\section{Materials and Methods}

Blood and tissue samples

Blood samples were collected from 40 patients (18 female and 22 male patients) with confirmed primary AML diagnosis (subtypes: M1, M2, M3, M4, M5 and 
M7) and from 10 healthy people. Patients were classified according to the French-American-British (FAB) diagnostic criteria. Informed consent was taken from all patients and healthy donors before inclusion into the study. Standard cytogenetic analysis was performed on patients' samples. The approval was obtained through the Investigation Review Board at Shahid Beheshti University of Medical Sciences. Normal testis sample was obtained from a prostate cancer patient following orchiectomy and was used as positive control for analysis of testis specific genes expression.

RNA extraction, cDNA synthesis, and quantitative realtime RT-PCR

Peripheral blood mononuclear cells were isolated from blood samples using Biosera Lymphosep, Lymphozyte separation media. Total RNA was isolated from leukocytes of patients using Tripure isolation reagent (Roche, Mannheim, Germany) according to the manufacturer's instructions. RNA was analyzed by Biowave II spectrophotometer (Biochrom Cambridge, England) to verify purity and concentration. Then, $1 \mu \mathrm{g}$ of RNA was used for cDNA synthesis by Fermentas RevertAidTM H Minus First Strand cDNA Synthesis Kit. Synthesized cDNA was analyzed spectrophotometrically to confirm concentration. Quantitative real time RT-PCR reaction was carried out on a rotor gene 6000 corbette detection system using AccuPower ${ }^{\circledR}$ 2X Greenstar qPCR Master Mix (BIONEER, USA). Thermal cycling conditions were: $5 \mathrm{~min}$ at $95^{\circ} \mathrm{C}, 40$ cycles of a denaturation step for $10 \mathrm{~s}$ at $95^{\circ} \mathrm{C}$ and a combined annealing/extension step for 30 $\mathrm{s}$ at $59^{\circ} \mathrm{C}$. Each run contained no template control (NTC) consisting of $\mathrm{H}_{2} \mathrm{O}$ for target and reference genes. Beta 2 microglobulin (B2M) gene was used as normalizer. Primer sequences can be provided via request. Melting curve analysis was done to validate specificity of PCR products. Real time RT-PCR products were also electrophoresed on $1.5 \%$ agarose gel to verify product sizes and specificity.

\section{Statistical analysis}

Fold changes in gene expression were determined by LinRegPCR(2) (Software for analysis of quantitative real-time PCR data) and Relative Expression Software Tool-RGC)-version 3 (Calculation Software for the Relative Expression in real-time PCR using Pair Wise Fixed Reallocation Randomization Test $^{\odot}$ ). The amounts of mRNA for each gene in the blood samples were standardized to the B2M mRNA as follows: $-\Delta \mathrm{CT}=-[\mathrm{CT}$ Gene of interest - CT B2M]. The level of probability was set at $\mathrm{p}<0.05$ as statistically significant.

\section{Results}

\section{Cytogenetic analysis}

Different cytogenetic abnormalities were seen in AML patients including $\mathrm{t}(8,21), \mathrm{t}(15,17), \mathrm{t}(16,16),+8,-5,-7$ with the first one being the most prevalent. About $60 \%$ of AML patients had normal karyotypes. No significant correlation was seen between cytogenetic abnormalities and gene expression.

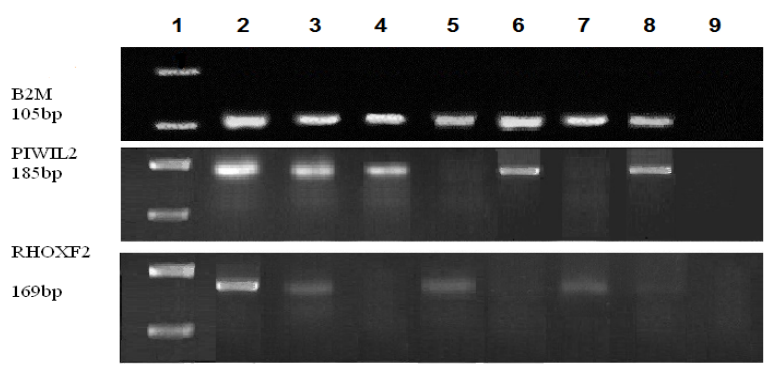

Figure 1. Genes Expression Results in Blood Samples: Lane 1: DNA Size Marker, Lane 2: Testis, Lanes: 3-4: Normal Samples, Lanes 5-8: AML Samples, Lane 9: Negative Control

RHOXF2 and PIWIL2 expression in normal lymphocytes

All 10 normal samples showed PIWIL2 expression, whereas 3 normal samples showed RHOXF2 expression (Figure 1).

\section{RHOXF2 and PIWIL2 expression in AML patients}

RHOXF2 expression was seen in 8 AML patients with similar distribution in both sexes. PIWIL2 expression was detected in 19 AML patients including 14 male and 5 female patients. Among those expressed PIWIL2, 10 had normal karyotypes. Five patients expressed both genes (Figure 1).

RHOXF 2 and PIWIL2 expression ratios in AML patients and normal donors

No significant difference was seen in the expression ratios of these 2 genes between AML patients and normal donors.

\section{Discussion}

Cancer stem cells are a subset of cells present in tumor bulks capable of self-renewal and the exclusive ability to reproduce malignant tumors indefinitely (Tabarestani and Ghafouri-Fard, 2012). The first cancer stem cells have been identified in AML. The detection of these cells has significant clinical implications especially to improve treatment outcome and prevent relapse of the disease (Chan and Huntly, 2008). In this study, we have evaluated the expression of two previously known stem cell markers (RHOXF2 and PIWIL2) in AML patients and normal donors. Previous data have indicated that both PIWIL2 and RHOXF2 have testis specific expression pattern. PIWIL2 has been shown to be expressed in various stages of breast cancers and cervical neoplasia, so proposed as a novel biomarker for these cancers (He et al., 2010; Liu et al., 2010). However, here we have shown for the first time that both PIWIL2 and PEPP2 are expressed in normal leukocytes. Some other genes have been believed to have testis specific or testis restricted expression but shown to be expressed in a variety of normal tissues. For instance, acrosin binding protein (ACRBP, OY-TES-1) was supposed to be expressed only in testis among normal tissues, and in a range of cancers such as bladder, breast, lung, liver, and colon cancers (Ono et al., 2001). It was at the center of attention for a period of time as cancer biomarker and target for immunotherapy. It has been also 
shown to be expressed in mesenchymal stem cells of bone marrow at both mRNA and protein levels (Cen et al., 2012). However, recent data indicate that it is expressed in a variety of normal tissues (CTDatabase: http://www.cta. lncc.br/index.php). The main advantage of using CT genes in immunotherapy resides in their restricted expression in normal tissues which diminishes treatment side effects. In addition, as testis is considered as an immune-privileged site, the absence of CT expression in normal tissues except for testis and strong expression in cancer tissues increase the probability of eliciting immune responses. The data presented here can put the significance of these 2 genes for cancer immunotherapy under question.

\section{Acknowledgements}

This study was supported by a grant from Shahid Beheshti University of Medical Sciences and done as a part of M.Sc. thesis.

\section{References}

Atanackovic D, Luetkens T, Kloth B, et al (2011). Cancer-testis antigen expression and its epigenetic modulation in acute myeloid leukemia. Am J Hematol, 86, 918-22.

Cen YH, Guo WW, Luo B, et al (2012). Knockdown of OY-TES-1 by RNAi causes cell cycle arrest and migration decrease in bone marrow-derived mesenchymal stem cells. Cell Biol Int, 36, 917-22.

Chan WI, Huntly BJ (2008). Leukemia stem cells in acute myeloid leukemia. Semin Oncol, 35, 326-35.

Costello RT, Rey J, Fauriat C, Gastaut JA, Olive D (2003). New approaches in the immunotherapy of haematological malignancies. Eur J Haematol, 70, 333-45.

Ghafouri-Fard S, Abdollahi DZ, Omrani M, Azizi F (2012). shRNA mediated RHOXF1 silencing influences expression of BCL2 but not CASP8 in MCF-7 and MDA-MB-231 cell lines. Asian Pac J Cancer Prev, 13, 5865-9.

Ghafouri-Fard S, Modarressi MH (2009). Cancer-testis antigens: potential targets for cancer immunotherapy. Arch Iran Med, 12, 395-404.

He G, Chen L, Ye Y, et al (2010). PIWIL2 expressed in various stages of cervical neoplasia is a potential complementary marker for p16. Am J Transl Res, 2, 156-69.

Lee JH, Schutte D, Wulf G, et al (2006). Stem-cell protein PIWIL2 is widely expressed in tumors and inhibits apoptosis through activation of Stat3/Bcl-XL pathway. Hum Mol Genet, 15, 201-11.

Lim SH, Zhang Y, Zhang J (2012). Cancer-testis antigens: the current status on antigen regulation and potential clinical use. Am J Blood Res, 2, 29-35.

Liu JJ, Shen R, Chen L, et al (2010). PIWIL2 is expressed in various stages of breast cancers and has the potential to be used as a novel biomarker. Int J Clin Exp Pathol, 3, 328-37.

Meklat F, Li Z, Wang Z, et al (2007). Cancer-testis antigens in haematological malignancies. Br J Haematol, 136, 769-76.

Mukda E, Pintaraks K, Sawangpanich R, Wiangnon S, Pakakasama S (2011). FLT3 and NPM1 gene mutations in childhood acute myeloblastic leukemia. Asian Pac J Cancer Prev, 12, 1827-31.

Shibata-Minoshima F, Oki T, Doki, N, et al (2012). Identification of RHOXF2 (PEPP2) as a cancer-promoting gene by expression cloning. Int J Oncol, 40, 93-8.

Ono T, Kurashige T, Harada N, et al (2001). Identification of proacrosin binding protein $\mathrm{sp} 32$ precursor as a human cancer/ testis antigen. Proc Natl Acad Sci USA, 98, 3282-7.

Siegel R, Naishadham D, Jemal A (2013). Cancer statistics, 2013. CA Cancer J Clin, 63, 11-30.

Tabarestani S, Ghafouri-Fard S (2012). Cancer stem cells and response to therapy. Asian Pac J Cancer Prev, 13, 5951-8.

Yazarloo F, Shirkoohi R, Mobasheri MB, Emami A, Modarressi MH (2013). Expression analysis of four testis-specific genes AURKC, OIP5, PIWIL2 and TAF7L in acute myeloid leukemia: a gender-dependent expression pattern. Med Oncol, 30, 368.

Yin DT, Wang Q, Chen L, et al (2011). Germline stem cell gene PIWIL2 mediates DNA repair through relaxation of chromatin. PLoS One, 6, 27154.

Zou Y, Zhong W (2012). A likely role for a novel PH-domain containing protein, PEPP2, in connecting membrane and cytoskeleton. Biocell, 36, 127-32. 